\title{
Genetics of structural and functional brain changes in autism spectrum disorder
}

\author{
Sheema Hashem', Sabah Nisar', Ajaz A. Bhat $\mathbb{D}^{1}$, Santosh Kumar Yadav ${ }^{1}$, Muhammad Waqar Azeem², Puneet Bagga ${ }^{3}$,
} Khalid Fakhro $\mathbb{E}^{4,5}$, Ravinder Reddy ${ }^{3}$, Michael P. Frenneaux ${ }^{6}$ and Mohammad Haris (1) ${ }^{1,7}$

\begin{abstract}
Autism spectrum disorder (ASD) is a neurological and developmental disorder characterized by social impairment and restricted interactive and communicative behaviors. It may occur as an isolated disorder or in the context of other neurological, psychiatric, developmental, and genetic disorders. Due to rapid developments in genomics and imaging technologies, imaging genetics studies of ASD have evolved in the last few years. Increased risk for ASD diagnosis is found to be related to many specific single-nucleotide polymorphisms, and the study of genetic mechanisms and noninvasive imaging has opened various approaches that can help diagnose ASD at the nascent level. Identifying risk genes related to structural and functional changes in the brain of ASD patients provide a better understanding of the disease's neuropsychiatry and can help identify targets for therapeutic intervention that could be useful for the clinical management of ASD patients.
\end{abstract}

\section{Introduction}

Autism spectrum disorder (ASD) is a neurological and developmental disorder consisting of a wide range of symptoms and disability that develop in early childhood and persists throughout life. The common symptoms of ASD include limited activities, lower engagement and communication, talking and learning problems, and repetitive behavior. According to the World Health Organization, the global burden of ASD is continuously growing, with a current prevalence rate of 1 in 160 children. Reported prevalence rates vary widely from country to country though. Recent data from the Centers for Disease Control and Prevention showed that about 1 in 68 children in the United States had been identified with some form of ASD, with more than 3 million people affected $^{1}$. A recent study estimated prevalence of ASD in the United States in 2014-2016 was 2.47\% among adolescents and children ${ }^{2}$. While in the United Kingdom, the

\footnotetext{
Correspondence: Mohammad Haris (mharis@sidra.org)

${ }^{1}$ Functional and Molecular Imaging Laboratory, Sidra Medicine, Doha, Qatar

2Department of Psychiatry, Sidra Medicine, Doha, Qatar

Full list of author information is available at the end of the article

These authors contributed equally: Sheema Hashem, Sabah Nisar
}

annual prevalence rate for children aged 8 years between 2004 and 2010 was 3.8/1000 for boys and $0.8 / 1000$ for girls $^{3}$. Recent studies have shown that the pooled ASD prevalence estimate in Asia is $0.36 \%$, including data from nine countries (China, Korea, India, Bangladesh, Lebanon, Iran, Israel, Nepal and Sri Lanka) ${ }^{4}$. The prevalence of ASD in the Middle East region was documented to be 1.4 per 10,000 children in $\mathrm{Oman}^{5}$, 4.3 per 10,000 children in Bahrain $^{6}, 1 / 167$ in Saudi Arabia ${ }^{7}$, and a recent study reported ASD prevalence to be $1.14 \%$ in children aged 6-11 years in Qatar ${ }^{8}$.

ASD incidence is $4-5$ times greater in males than in females ${ }^{9}$. The exact cause of ASD remains unclear; however, it is thought that both genetic and environmental factors play essential roles. The effect of ASD on society is enormous and multifaceted as it affects not only the child but also the siblings and parents and significantly disturbs the functioning of family routine life. Individuals with ASD are very likely to encounter the criminal justice system, mostly due to a lack of knowledge of their social and communication difficulties. There are also financial pressures associated with the recovery and decreased opportunities for jobs. Various studies have focused on 
the problems faced by families of autistic children ${ }^{10}$. It may affect the performance of the child at school, based on the severity of the disorder, and if untreated or undiagnosed, it may continue into adulthood, affecting both personal and professional life. Although there is no standard treatment for ASD, early detection and use of successful therapies can make a difference in the management and improvement of symptoms, and thus the overall quality of life of children with ASD. Before the clinical symptoms occur, therefore, a consensus is reached in treating ASD based on early detection.

Recent cohort studies have reported that hereditary factors can be traced to more than $50 \%$ of autism cases ${ }^{11}$. Historically, the genetic essence of ASD has been identified in family and twin studies ${ }^{12,13}$. The study of family exome sequencing has found some rare de novo mutations in people with ASD as opposed to healthy controls. It is estimated that $\sim 1000$ genes are involved in autism ${ }^{14,15}$. On the other hand, the main variants associated with autism ${ }^{16}$ are yet to be identified in genome-wide association studies. By understanding the evidence at gene expression, we will obtain a complete understanding of autism which can aid in the clinical treatment of patients with ASD.

On the other hand, noninvasive imaging provides information on the structural and functional changes of autism in the brain. Studies have shown that connecting genomics to imaging can provide a better understanding of ASD's pathophysiology ${ }^{17}$ and can help develop therapeutic approaches to prevent or reverse ASD brain changes. In this review article, as demonstrated in the imaging, we illustrate how genetic variations are correlated with the structural and functional changes in the brain.

\section{Changes in the autistic brain Pathological findings}

Postmortem studies conducted on autistic brains helped to identify the neuroanatomical changes associated with ASD. Smaller cell size and increased density of cells in the hippocampus, limbic system, entorhinal cortex, and amygdala have been observed in all ages of ASD individuals ${ }^{18}$. Young patients with autism showed abnormally enlarged neurons in the cerebellar nuclei, inferior olive, and vertical limb of the diagonal band of broca ${ }^{18}$. Another postmortem study found an increased number of neurons in the prefrontal cortex in the brain of ASD children ${ }^{19}$. Purkinje cell count was found to be decreased in the archicerebellar and neocerebellar cortices of the cerebellar hemisphere in autistic patients ${ }^{20}$. Another study observed a reduced number of small pale neurons in the brains of adult autistic patients ${ }^{21}$. A postmortem study of the brain of patients with ASD also reported altered axonal density and the presence of impaired myelin in white matter $(\mathrm{WM})^{22}$. Overall, the pathological findings suggest a restricted brain development pattern in ASD patients.

\section{Structural brain changes}

Brain changes in ASD patients have been primarily studied using magnetic resonance imaging (MRI). MRI is the most widely used noninvasive modality to examine the structural and functional brain changes in different neurological and neurodegenerative disorders. Various MRbased methods are applied to study the morphological, functional, and metabolic changes in the brain of ASD. MRI findings in children with ASD ranging from 2 to 5 years of age revealed that there is an abnormal development of frontal and temporal lobes, lower gray matter (GM), WM, and amygdala volume compared to same-age healthy controls ${ }^{23}$. A comparison of the brain morphometric features in 3-4 years old ASD children with typically developing (TD) children and developmentally delayed children ${ }^{24}$ was conducted using high-resolution 3-D MRI. Increased cerebellar volume was found in children with ASD compared to TD, and developmentally delayed children and amygdala enlargement with increased cerebral volume was seen in ASD children ${ }^{24}$. Another study observed cerebral enlargement in both boys and girls with ASD compared to controls, and boys with regressive autism showed more structural alterations as compared to girls ${ }^{25}$. Volumetric MR studies demonstrated a 5-10\% increase in brain volume of ASD children who were scanned between 18 months and 4 years ${ }^{23,26}$.

Imaging studies have observed both decreased and increased cortical thickness in patients with ASD. One study, performed on eight autistic subjects, showed decreased cortical thickness in the regions of inferior frontal, occipital, supramarginal, and post- and precentral gyrus, anterior cingulate, prefrontal, parietal, and temporal cortex ${ }^{27}$. Another study showed increased cortical thickness in the parietal and temporal lobes of autistic children compared to healthy controls ${ }^{28}$. An MRI study of adults with ASD, who had delayed language development, also called high-functioning autism (HFA), observed increased cortical thickness in frontal, occipital, temporal, parietal, cingulate, and fusiform gyri while decreased cortical thickness was observed in the post- and paracentral gyrus ${ }^{29}$. Surface-based morphometry (SBM) analysis is also used to measure cortical thickness in ASD patients. An SBM based study found decreased cortical thickness in the left orbitofrontal and parahippocampal gyrus, left frontal pole, and pars triangularis, while increased cortical thickness was observed in the left precuneus and anterior cingulate $\operatorname{cortex}^{30}$. Another SBM based study reported reduced cortical volume in the left middle temporal gyrus, reduced gyrification index in the left supramarginal gyrus and increased cortical thickness in pars opercularis of inferior frontal gyrus in HFA compared to TD individuals ${ }^{31}$. The SBM approach was also used to compare the cortical abnormalities in individuals with low-functioning autism (LFA) and HFA and 
Asperger's syndrome with TD controls. In HFA individuals, shape abnormality was observed bilaterally in parietal operculum and ventral postcentral gyrus. LFA individuals showed shape abnormality in the pars opercularis located in the inferior frontal gyrus, and individuals with Asperger's syndrome showed bilateral abnormalities in the intraparietal sulcus region ${ }^{32}$. Voxelbased morphometry analysis observed increased GM volume in frontal, parietal, temporal lobes and limbic system and reduced WM volume in limbic system, frontal and temporal lobes of autistic subjects ${ }^{33}$. Another voxelbased morphometry study showed reduced GM volumes in frontal, temporal, and parietal lobes in HFA individuals compared to healthy controls ${ }^{34}$.

Diffusion tensor imaging (DTI), a noninvasive MRI technique, exploits the diffusion properties of a water molecule to map the microstructural, architectural, and compositional characteristics of the tissue. Fractional anisotropy (FA) and mean diffusivity are two commonly used DTI metrices in mapping the restricted movement of water molecules in the tissue, where higher FA associates with the greater directionality and tissue integrity ${ }^{35}$. DTI is widely used to track the changes in the microstructural properties of WM such as axonal density, axonal injury, and myelination in various human brain' disorders. Provided that ASD is a developmental disorder, DTI can be used to identify variations in WM to better define developmental trajectories in individuals with $\mathrm{ASD}^{36}$. Increased FA was observed in the frontal lobes and corpus callosum regions of children aged 2-3 years with ASD compared to healthy controls ${ }^{37}$. While, another study showed reduced FA in frontal-posterior tracts in the brain of children with ASD, aged 10-18 years ${ }^{38}$. DTI studies showed a difference in trajectories of FA in infants aged 6-24 months who were at higher family risk of developing ASD. FA was higher in infants who had ASD diagnosis at the age of 6 months, while FA was found to be lower at the age of 24 months $^{39}$. One study reported decreased FA in the parietal lobes, temporal lobes, lateral occipital cortex, left anterior cingulate, middle frontal cortex, and in the corticospinal tract of individuals with HFA as compared to the controls ${ }^{34}$. DTI tractography confirmed abnormality of the WM structures in the regions of the corpus callosum, inferior longitudinal and fronto-occipital fasciculus, and superior longitudinal fasciculus in ASD patients ${ }^{40}$.

\section{Genes affecting brain morphology \\ Preclinical studies}

While there may be several specific genes affected in autism, there is increasing evidence that these genes converge on a limited number of biological pathways that include changes in cortical development, synapse function, transcription and translation, chromatin modification, and microglial activation ${ }^{41,42}$ (Table 1).
Neuroligin-3 (NL3) is a major cell adhesion protein that plays a vital role in the development of the synapse, and it has also been implicated in ASD. An ex vivo MRI study showed a reduction in various WM and GM regions of $N L 3$ knockin mice, with deficits in social and anxietyrelated behaviors ${ }^{43}$. A similar study used MRI to image mouse models with mutations implicated in autism, such as NL3 knockin, MECP2, and integrin $\beta 3$ (ITGB3) homozygous knockout mouse models to investigate the effects of these mutations on brain morphology ${ }^{44}$. The study found enlarged WM, and GM volumes of crus II lobule in NL3 mutants increased cerebellar volumes in $M E C P 2$ mutants and reduced cerebellar structures in ITGB3 mutants ${ }^{44}$. A neuroimaging study reported reduced total brain volume and hippocampal size with enlarged basal ganglia structures in SHANK3 knockout mice compared to a prenatal zinc-deficient mouse model of $\mathrm{ASD}^{45}$.

A recent study on mice with ANK2 mutations showed an increased number of excitatory synapses during postnatal development with increased axonal branching, which supports the presence of altered connectivity and penetrant behavioral impairments in mice and humans carrying ASD-related ANK2 mutations ${ }^{46}$. Mutations in CHD2 have been linked with various neurodevelopmental disorders, including $\mathrm{ASD}^{47}$. CHD2 is shown to play an essential role in brain development as the suppression of CHD2 can inhibit the self-renewing ability of radial glial cells and can increase the generation of intermediate progenitor cells and neurons during the process of neurogenesis that might contribute to abnormal neurodevelopment ${ }^{48}$. Another study showed that $C H D 2$ knockdown zebrafish exhibited altered locomotor activity, which contributed to epileptic encephalopathy ${ }^{49}$. These studies show that there is a genetic overlap found between ASD disorder and other neurological disorders such as schizophrenia, epilepsy, and $\mathrm{ID}^{50}$. In addition, knockdown of CHD8 resulted in reduced axon and dendritic growth with delayed neuronal migration in mice ${ }^{51}$. CHD8 suppression in zebrafish affected the gene expression in neurodevelopmental pathways related to the proliferation of neural progenitor cells ${ }^{52}$.

One of the studies showed that mice mutants of HOXA1/HOXB1 had several defects, including the loss of rhombomere 4 and 5 and the loss of 2nd branchial arch affecting the development of the hindbrain ${ }^{53}$. Previous studies have shown the role of $16 p 11$ deletions ${ }^{54}$ and the CHD8 gene ${ }^{55}$ in the brain overgrowth in idiopathic ASD. The postnatal development mechanisms such as pruning and dendritic arborization are affected by the overproliferation of cortical progenitor cells. The overproduction of neocortical neurons also contributed to the autistic-like features in mice ${ }^{56}$. Studies have reported that $16 p 11$ deletions resulted in the alteration of cortical progenitor cell proliferation in mice and increased brain 


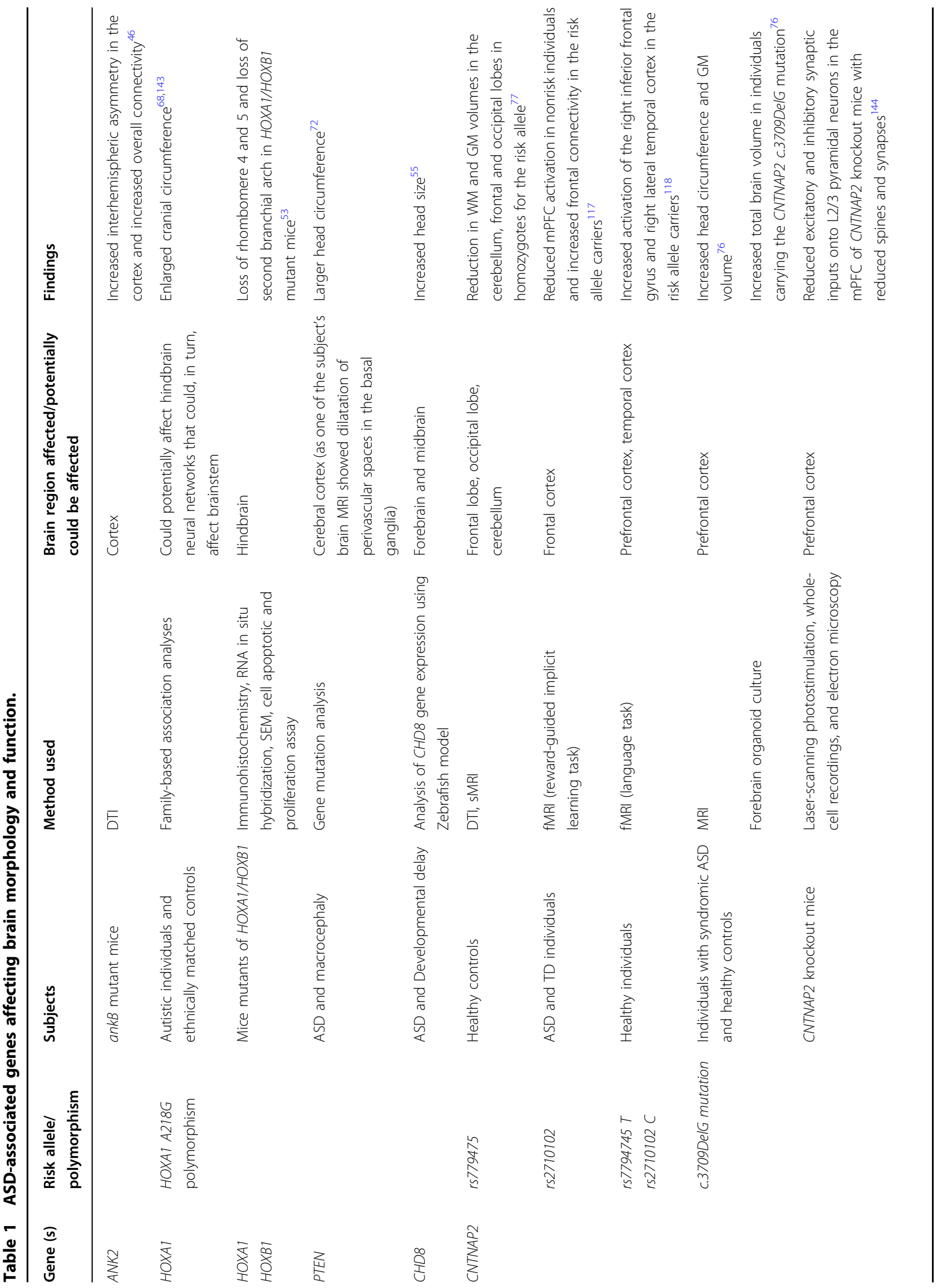




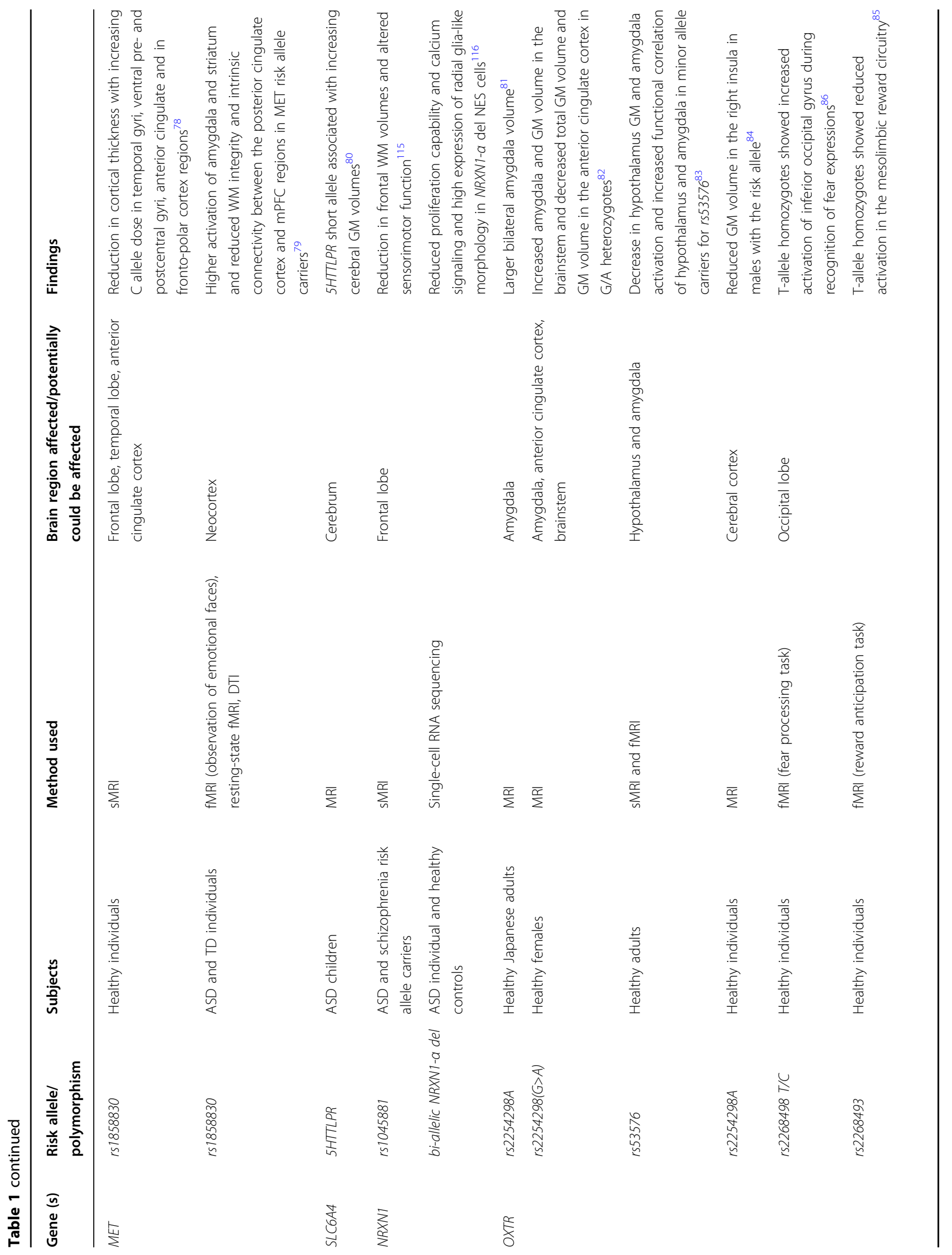




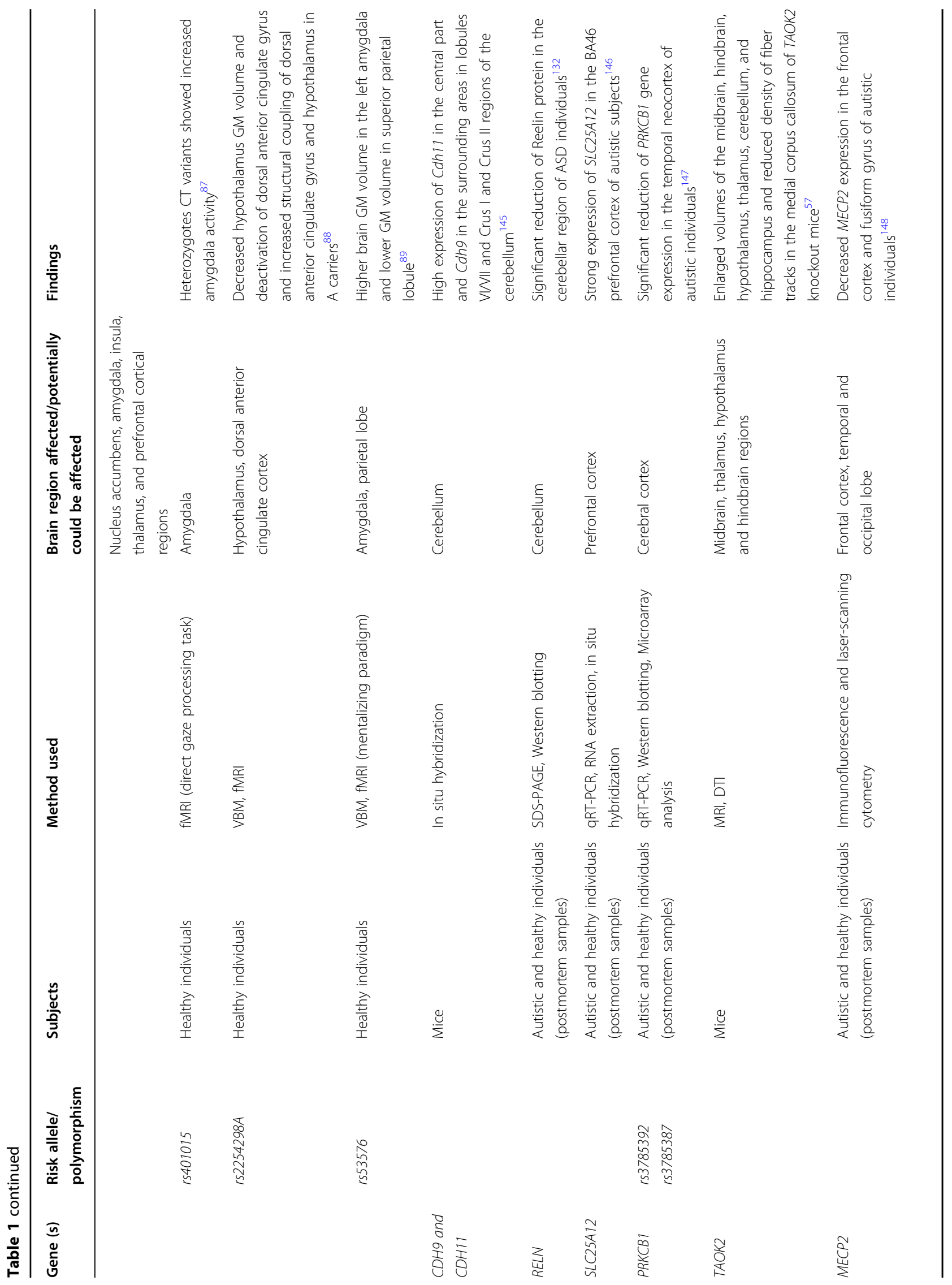




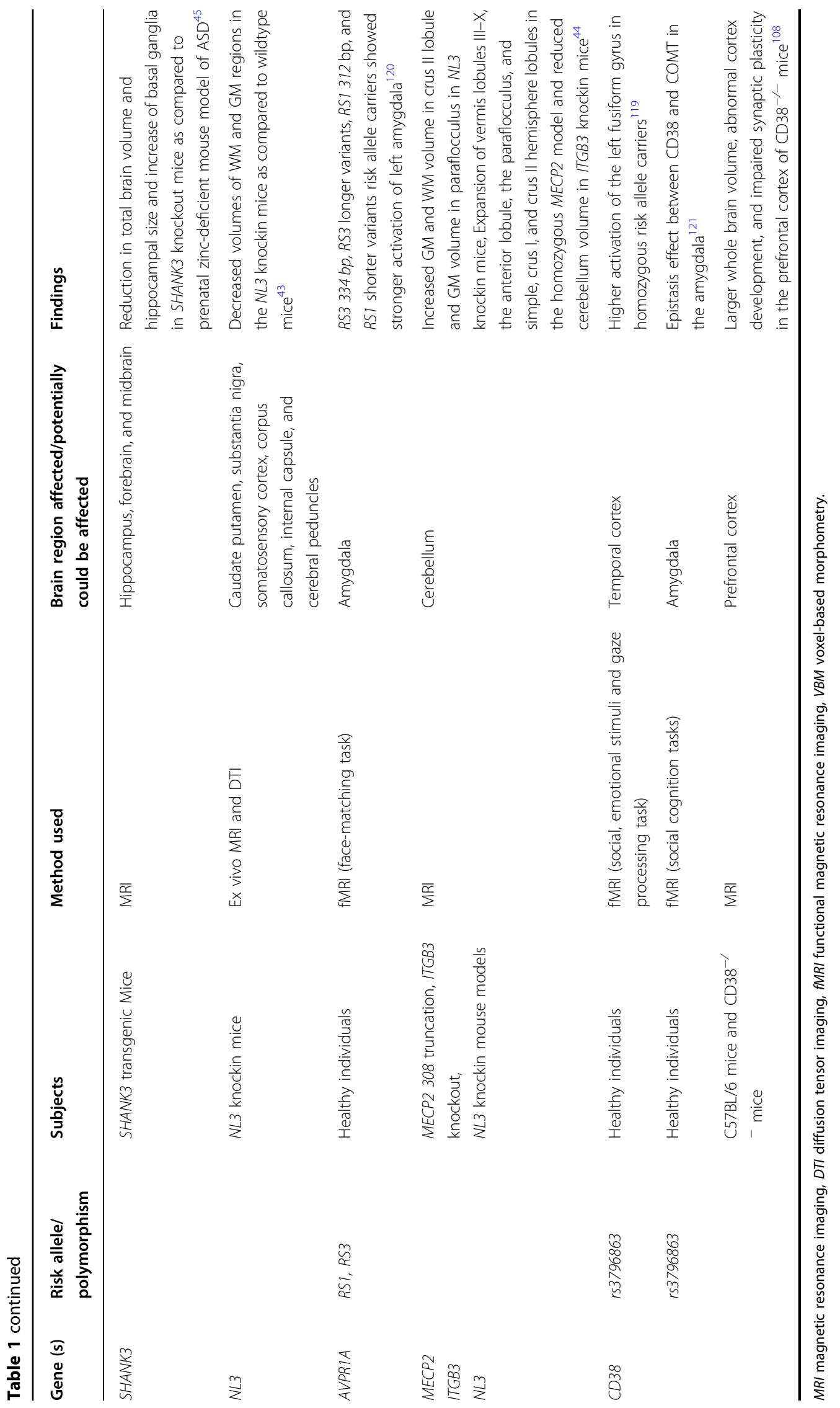


HOX genes play an important role in developmental pathways and affect the development of Hindbrain

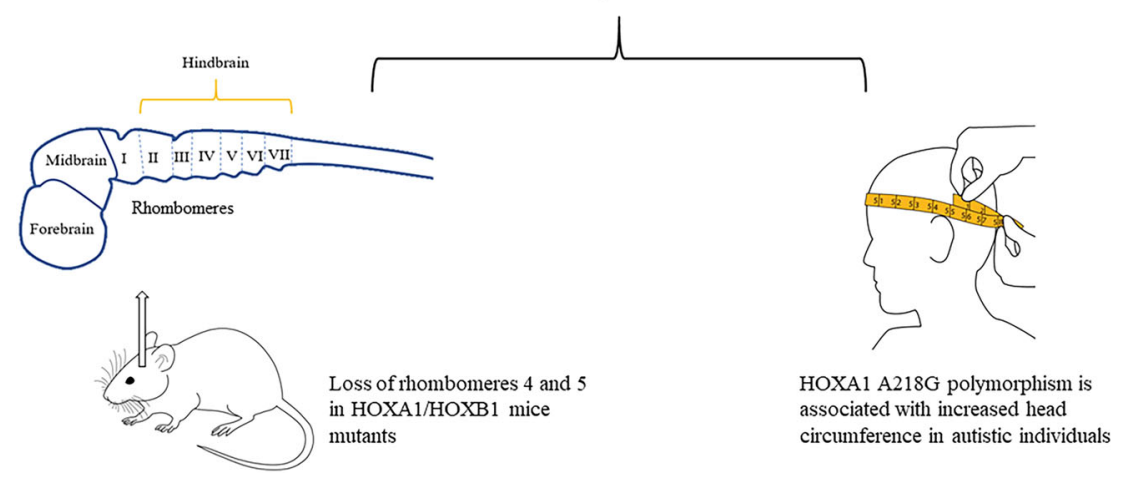

Fig. 1 Structural brain changes associated with HOX genes. HOXA1 A218 gene polymorphism is associated with an increased head circumference in autistic individuals, and HOXA1/HOXB1 mice mutants showed loss of rhombomeres 4 and 5 affecting the hindbrain development ${ }^{53,68}$.

volumes in individuals with syndromic $\mathrm{ASD}^{54}$. One of the genes associated with the $16 p 11.2$ region known as TAOK2 has been found to cause ASD-related cognitive abnormalities $^{57}$. A neuroimaging study found dosagedependent abnormalities in TAOK2 heterozygous and knockout mice. MRI of the TAOK2 knockout mice revealed enlarged midbrain, hindbrain, hypothalamus, thalamus, cerebellum, and hippocampus volumes, with a regional delay in the development of the neuronal track in the medial corpus callosum ${ }^{57}$.

Animal studies have shown that the MET protein regulates early cortical development in the neurobiology of ASD. A study by Qiu et al. showed excitatory hyperconnectivity in neocortical circuits of Met conditional knockout mice ${ }^{58}$. While another study showed that the reduction of MET and HGF/SF correlated with calbindin + interneurons in the cortex of urokinase-type plasminogen activator receptor knockout mouse, thereby affecting cortical development ${ }^{59}$.

\section{Clinical studies}

In the clinical setting, several imaging studies were conducted that link specific gene polymorphisms with abnormal brain morphology in ASD patients (Table 1). Gene deletions such as $22 q 11.2$ and $16 p 11.2$ are associated with ASD. These deletions are also found to be linked with other neuropsychiatric disorders, including DiGeorge Syndrome, conotruncal anomaly face syndrome, velocardiofacial syndrome, intellectual disability, and schizophrenia ${ }^{60,61} .22 q 11.2$ deletion in patients having high-risk negative symptoms at risk of developing schizophrenia showed decreased gyrification mainly in the medial occipital and temporal regions, involved in social cognition and early visual processing ${ }^{62}$. Another study reported increased cortical thickness in the frontal lobe, cerebral cortex, and superior parietal lobes and decreased cortical thickness in the regions of superior temporal gyrus and posterior cingulate cortex in patients with $22 q 11.2$ deletion syndrome ${ }^{63}$.

Head circumference is abnormally increased in ASD patients ${ }^{64}$. Many factors can be responsible for the brain overgrowth in ASD, such as altered synapses during the development process, increased neurites or the number of neurons in the brain. $H O X$ genes play an essential role in the development of organs and are responsible for the proper positioning of organism's segment structures during embryonic development (Fig. 1). Out of all the HOX genes, HOXA1 is a critical gene that is involved in the development of the central nervous system, internal ear, and hyoid bones ${ }^{65,66}$. HOXA1 polymorphism has been found to induce an increased head growth rate in autistic and TD children ${ }^{67}$. In addition, autistic individuals with HOXA1 A218G polymorphism displayed an enlarged cranial circumference ${ }^{68}$, while in autistic patients, one study reported minor contributions of the $H O X B 1$ gene to head circumference ${ }^{69}$.

Mutations in PTEN have also been associated with brain morphological changes in ASD. PTEN mutations have been observed in Cowden syndrome, and macrocephaly is the main feature of patients with Cowden syndrome, and some of the individuals with this syndrome are also found to be autistic ${ }^{70,71}$. Sequencing of the PTEN gene in ASD patients revealed three heterozygous germline mutations $^{72}$ and a missense mutation ${ }^{73}$. An ASD-related syndrome, known as cortical dysplasia-focal epilepsy (CDFE) is a rare neuronal migration disorder, and patients with CDFE exhibit autistic characteristics. A study reported a homozygous mutation in the CNTNAP2 gene in all CDFE patients, which is responsible for an early developmental insult, mainly in the frontal and temporal neocortex ${ }^{74}$. Another study showed that the impact of CNTNAP2 missense variants on axonal growth as the loss of 
CNTNAP2 allele contributes to the disruption in axonal growth $^{75}$. An MRI study investigated the homozygous c.3709DelG mutation in CNTNAP2 in the forebrain organoids generated from human induced pluripotent stem cells that were derived from patients with syndromic ASD. The study found that individuals with c.3709DelG mutation in CNTNAP2 displayed increased head circumference and increased GM volume ${ }^{76}$. An imaging genetic study found an association of CNTNAP2 rs779475 single-nucleotide polymorphism with WM and GM morphology in healthy controls and observed reduced volume of GM in the regions of frontal and occipital lobes and cerebellum. However, there was a reduction in the WM volume in the regions of right rostral cingulum, right caudal inferior fronto-occipital fasciculus, and the posterior thalamic radiations in homozygotes for the risk allele (rs779475 T/T) as compared to the nonrisk homozygotes and a group of $r s 779475 T$ heterozygotes ${ }^{77}$.

Mutations in the chromodomain helicase DNA binding protein $8(C H D 8)$ gene, which plays a vital role in the chromatin remodeling process, are amongst the most commonly reported mutations in ASD. CHD8 mutations have also been linked with axonal and dendritic growth, and a study showed that CHD8 is highly expressed in a microtubule-associated-protein 2 positive neurons and parvalbumin neurons which plays an essential role in the development of neocortex in the human brain. The findings of the study suggested that CHD 8 mutations in humans can result in neuronal deficits that can contribute to ASD pathophysiology ${ }^{51}$.

Studies have proposed the association of MET protooncogene, receptor tyrosine kinase (MET), as a candidate risk gene for ASD. In order to find the association of MET with cortical development, a structural MRI study investigated the relationship between a single-nucleotide polymorphism within the MET promoter (rs1858830, $G \rightarrow C$ ) and the development of cortical thickness in ASD individuals. The study observed cortical thickness reduction in the regions of ventral precentral and postcentral gyri, superior and middle temporal gyri, anterior cingulate cortex, and in the right fronto-polar cortex with increasing $\mathrm{C}$ allele dose ${ }^{78}$. Another study found that MET risk allele carrier (rs1858830) individuals with ASD and TD showed higher activation of amygdala, reduced WM integrity, and reduced connectivity between the posterior cingulate cortex and medial prefrontal cortex $(\mathrm{mPFC})^{79}$.

A common polymorphism in the serotonin-transporter gene (SLC6A4) involving a serotonin-transporter related polymorphic region (5HTTLPR) results in the generation of short or long alleles. A study by Wassink et al. found that the 5HTTLPR short allele significantly influences the cortical GM volumes in male autistic children ${ }^{80}$. Several neuroimaging studies have investigated the effects of different oxytocin receptor $(O X T R)$ risk alleles (rs2254298, rs53576, rs226849, rs401015) on brain morphological changes in healthy adults ${ }^{81-89}$. OXTR risk allele carriers (rs2254298) showed increased amygdala volume $^{81,82,89}$, decreased GM volume in the hypothalamus $^{83}$, and right insula ${ }^{84}$. A study showed that dysregulation in the $\beta$-catenin/BRN2 transcriptional cascade was associated with an increased number of neurons and proliferation of neural progenitor cells derived from pluripotent stem cells of ASD individuals and also contributed to increased brain volumes ${ }^{90}$.

\section{Functional brain changes}

Resting-state fMRI (rsfMRI) is a powerful tool that is being used for mapping the neuronal activity of various neurological disorders. rsfMRI studies have revealed abnormalities in the neuronal network in ASD patients that help in understanding the underlying neurobiology of ASD. An rsfMRI study observed altered functional connectivity between the cerebral cortex and dentate nucleus in individuals with ASD, which is suggestive of impaired interaction between the cerebellum and social cortical regions of the brain ${ }^{91}$. Another study observed reduced integration of the default mode network with the mPFC and angular gyrus regions in the ASD group ${ }^{92}$. The mPFC region is mainly involved in decision making, and one of its primary function is encoding task-related information in the working memory ${ }^{93}$, while angular gyrus is involved in semantic processing, default mode network, social cognition, and memory retrieval and attention ${ }^{94}$. Another rsfMRI study showed that the underconnectivity in the temporal sulcus, which is a human voice processing region, in ASD children in addition to a decreased connectivity in reward-related regions in the brain of ASD children. While task-based fMRI showed altered network connectivity in multiple brain regions that control social and emotional processing in $\mathrm{ASD}^{95}$. A sentence comprehension task-based study showed decreased connectivity in cortical areas of ASD individuals, which signifies the low congruence of brain regions that are essential for integrating information ${ }^{96}$. Other task-based studies have also reported decreased connectivity in the fronto-parietal regions that are involved in working memory (n-back working memory task) ${ }^{97}$, facial processing (emotional facial processing task) ${ }^{98}$, response inhibition (simple inhibition/1-back inhibition task) ${ }^{99}$ and visuomotor coordination (measuring BOLD signal) ${ }^{100}$ in ASD individuals. Task-based study based on social processing found hypoactivation in social brain nodes in the regions of the $\mathrm{mPFC}$, frontal gyrus, insula, temporal sulcus, amygdala, and the fusiform gyrus ${ }^{101}$.

Task-based fMRI studies focused on cognitive control reported fronto-striatal dysfunction in ASD, specifically in frontal gyrus, basal ganglia, and anterior cingulate cortex regions. Task-based studies that involved examining brain 
responses associated with figures of speech like puns ${ }^{102}$ and examining the neural activity during the detection of senseless sentences ${ }^{103}$ have also shown communication impairments such as decreased brain synchrony in language processing regions in $\mathrm{ASD}^{102,103}$. Task-based studies focusing on reward processing showed impairments in mesolimbic and meso cortical brain regions in ASD. Both over connectivity and underconnectivity between frontal and posterior regions was observed in task-based functional connectivity study ${ }^{104}$. An fMRI study revealed that ASD individuals had reduced activation of higherorder social (fusiform, cingulate, amygdala, gyrus, insula) and executive processing (prefrontal cortex) of the brain and higher activation of lower-order structures that are involved in the mediation of primary motor and sensory processing during emotional task performance ${ }^{105}$. While some task-based studies such as source recognition $\operatorname{task}^{106}$ and emotional face processing task ${ }^{107}$ have reported increased connectivity between the brain regions of ASD individuals ${ }^{106,107}$.

\section{Limitations of functional imaging in ASD}

Neuroimaging can help bridge the gap between genes, environment, and different ASD behavioral phenotypes. Although functional imaging techniques such as fMRI have revolutionized the understanding of various mental disorders, the fMRI technique also contains several limitations. The big challenge in current fMRI-based methods is the impact of age/gender and the development of scanning techniques for the diagnosis of ASD across heterogeneous populations. One of the limitations of fMRI is that it has no role in measuring the direct neuronal activity instead it provides changes in the signal based on the blood oxygen level, which may lead to the false-positive or -negative signal specially in patients with abrupt behavioral changes such as ASD. Secondly, an individual's performance on a specific task must be considered in a task-based fMRI and must be well-thoughtout when analyzing the fMRI data. The fMRI data are also analyzed as group averages; therefore, it is not straightforward to diagnose individual autistic patients. This could be par by introducing an individualized diagnostic model based on artificial intelligence.

Furthermore, the data obtained from fMRI may be compromised to some extent due to head motion artifacts that alter voxel and stable state magnetization leading to distortion of anatomical locations and may show false regions associated with brain activity. In addition, fMRI data can only demonstrate that a particular region of the brain is involved in a specific cognitive function but cannot determine whether it is a cause or a consequence of that specific cognitive function. The lack of longitudinal data also makes it challenging to identify the abnormal developmental course in ASD during infancy.
Nevertheless, the integration of multiple sites and data sources and the increase in the number of subjects and intra-variability among subjects can improve the feasibility of the fMRI technique in ASD.

\section{Genes affecting brain functions \\ Preclinical studies}

Reduced levels of oxytocin have been found in ASD patients, and CD38 is a vital enzyme that controls oxytocin production. A CD $38^{-1-}$ mice study showed abnormal development of the cortex and impaired synaptic plasticity in the prefrontal cortex region with impaired social and emotional responses ${ }^{108}$ (Table 1). Genetic variations in the $1 \mathrm{~A}$ gene of the arginine vasopressin receptor (AVPR1A) were linked to autism. Impaired social recognition and reduced anxiety behaviors were observed in knockout mice of $A V P R 1 A$, while overexpression of AVPR1A in mice led to increased social memory ${ }^{109,110}$. There is also an inversion of cortical layering due to neuronal migration defects in the Reelin $(R E L N)$ knockout mice $^{111}$, resulting in various behavioral abnormalities, such as aggressive behavior and memory and learning impairments $^{112}$. CNVs of the cytoplasmic FMR1interacting protein 1 (CYFIP1) was found to be associated with ASD and other neuropsychiatric disorders such as schizophrenia. CYFIP1 plays an essential part in regional brain connectivity and corpus callosum function. A study found that the CYFIP1-heterozygous mice displayed reduced bilateral functional connectivity across the entire brain and defects in the WM architecture. In these mice, reduced myelination is also observed in the callosal axons, an altered presynaptic feature that contributes to the motor and sensorimotor abnormalities ${ }^{113}$.

Another rsfMRI study showed decreased local and longrange functional connectivity in prefrontal and midbrain of mice with the homozygous loss of CNTNAP2 and may contribute to the development of autistic-like features and other neurodevelopment disorders ${ }^{114}$.

\section{Clinical studies}

Imaging genetics studies have shown the involvement of ASD risk genes in altering the brain circuits that control reward and language processing, and social behavior (Table 1). Neurexin-1 (NRXN1) is one of the ASD risk genes that are found to influence the brain structure and functions, and its polymorphisms are found to be associated with structural alterations in the prefrontal-thalamic circuitry in healthy individuals, conferring a potential risk of developing ASD or schizophrenia ${ }^{115}$. Single-cell analysis of neural stem cells from an autistic patient with bi-allelic NRXN1- $\alpha$ deletion observed a phenotypic shift toward radial glia-like cell with impaired maturation action potential and reduced calcium signaling ${ }^{116}$. On the other hand, CNTNAP2 polymorphisms are found to be associated with altered brain 


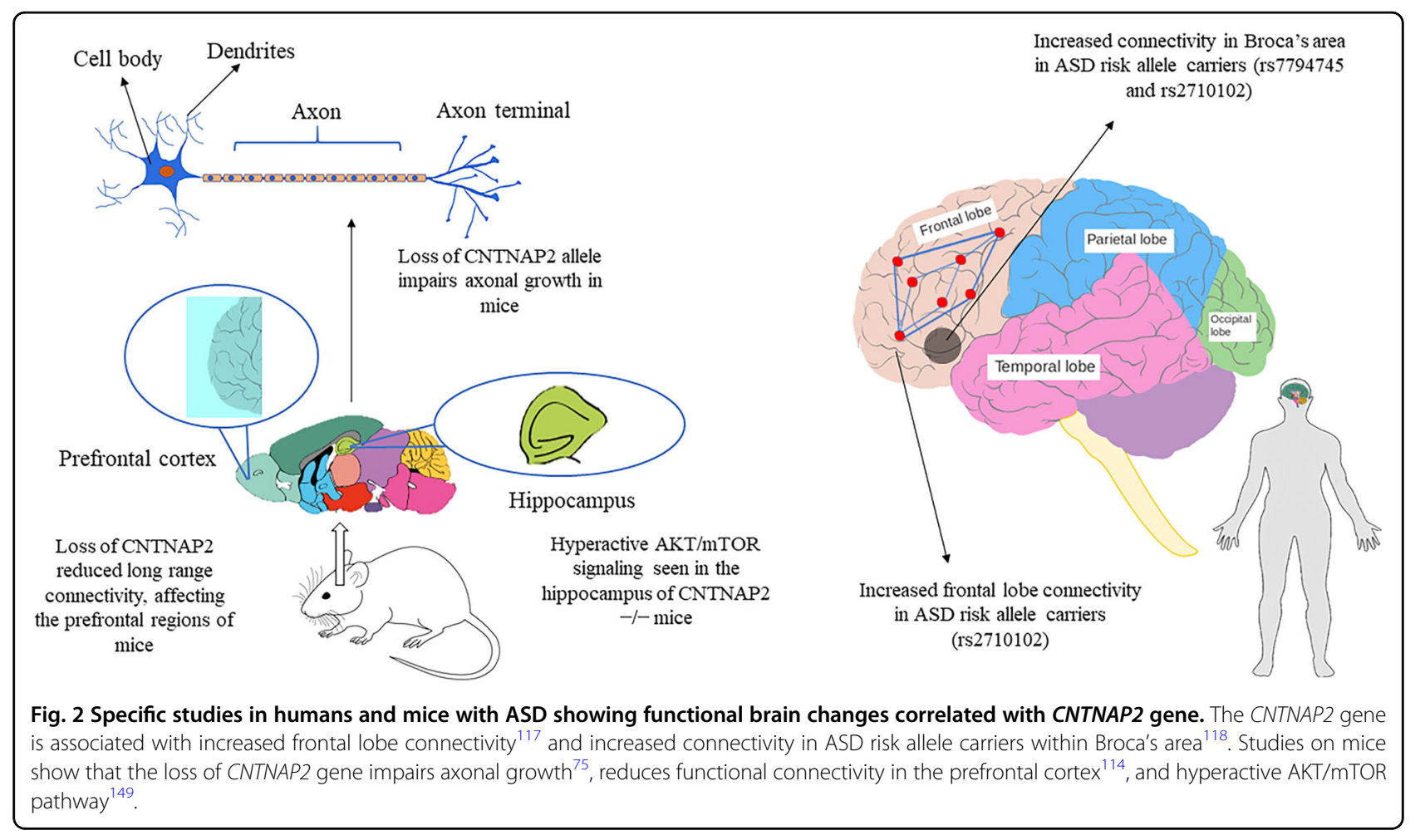

connectivity in regions that are involved in reward and language development ${ }^{117}$ (Fig. 2). Healthy individuals carrying the risk alleles for ASD and language impairment (rs7794745 T, rs2710102 C) showed increased activation in the right inferior frontal gyrus and right lateral temporal cortex $^{118}$. Mutations in the genes involved in the oxytocin/ vasopressin system may also contribute to ASD risk as they influence the function of brain regions such as the amygdala and hypothalamus that are associated with emotional and social processing ${ }^{119,120}$ (Fig. 3). Studies have found an association between a common genetic variant in $C D 38$ (rs3796863), autism, and the impact of oxytocin levels on brain response to social stimuli ${ }^{119,121}$. An fMRI study observed increased activation of the left fusiform gyrus in homozygotes for $C D 38$ genetic variant $r s 3796863^{119}$. The $M E T$ gene variants are also found to be involved in altering the connectivity and integrity of WM in the temporoparieto-occipital regions that are involved in high neurological functions such as working memory, language processing, and face and object recognition, all that may predispose an individual to $\mathrm{ASD}^{79}$. Another study reported that the loss of CNTNAP2 contributed to the reduction in long-range connectivity in the brain regions of mice, mainly affecting the prefrontal regions that act as connectivity hubs in the brain ${ }^{122}$ and this hypoconnectivity in the frontoposterior region lead to impaired social behaviors, predisposing to autism risk ${ }^{114}$. The OXTR polymorphism rs1042778 is found to be associated with two common clinical phenotypes of ASD, such as aggression and panic in male ASD patients. As compared to GG homozygotes, the $T$-allele carriers were at higher risk of possessing aggressive and panic behaviors ${ }^{123}$. Some of the task-based fMRI studies in healthy individuals carrying the OXTR risk alleles showed increased activation of inferior occipital gyrus during a fear processing task ${ }^{86}$, decreased activation in the mesolimbic reward circuitry in a reward anticipation task ${ }^{85}$ and increased amygdala activity during a direct gaze processing task ${ }^{87}$. One study found elevated levels of AVP with reduced levels of apelin in ASD patients that point to the vasopressinergic dysfunction in autism ${ }^{124}$. Another study proposed that the shorter alleles of $R S 1$ polymorphism lead to reduced transcription of $A V P R 1 A$ that may increase the susceptibility to ASD ${ }^{125}$. An fMRI study found an association between ASD and AVPR1A genetic variants and reported differential activation of amygdala during a facematching task in $R S 1$ and $R S 3$ risk allele carriers ${ }^{120}$.

Reduced expression of three genes namely metaxin 2 (MTX2), neurofilament light polypeptide (NEFL), and solute carrier family 25, member 27 (SLC25A27) was found in the anterior cingulate gyrus, the motor cortex, and thalamus regions of autistic patients. NEFL plays a vital role in the maintenance and assembly of the axonal cytoskeleton $^{126}$, while SLC25A27 is expressed in the central nervous system and has been found to play an essential role in neuronal cell differentiation ${ }^{127}$, apoptosis inhibition ${ }^{128}$, and reduction of reactive oxygen species ${ }^{129}$.

One of the critical regulators of neuronal migration is RELN glycoprotein, shown to play a vital role in the 


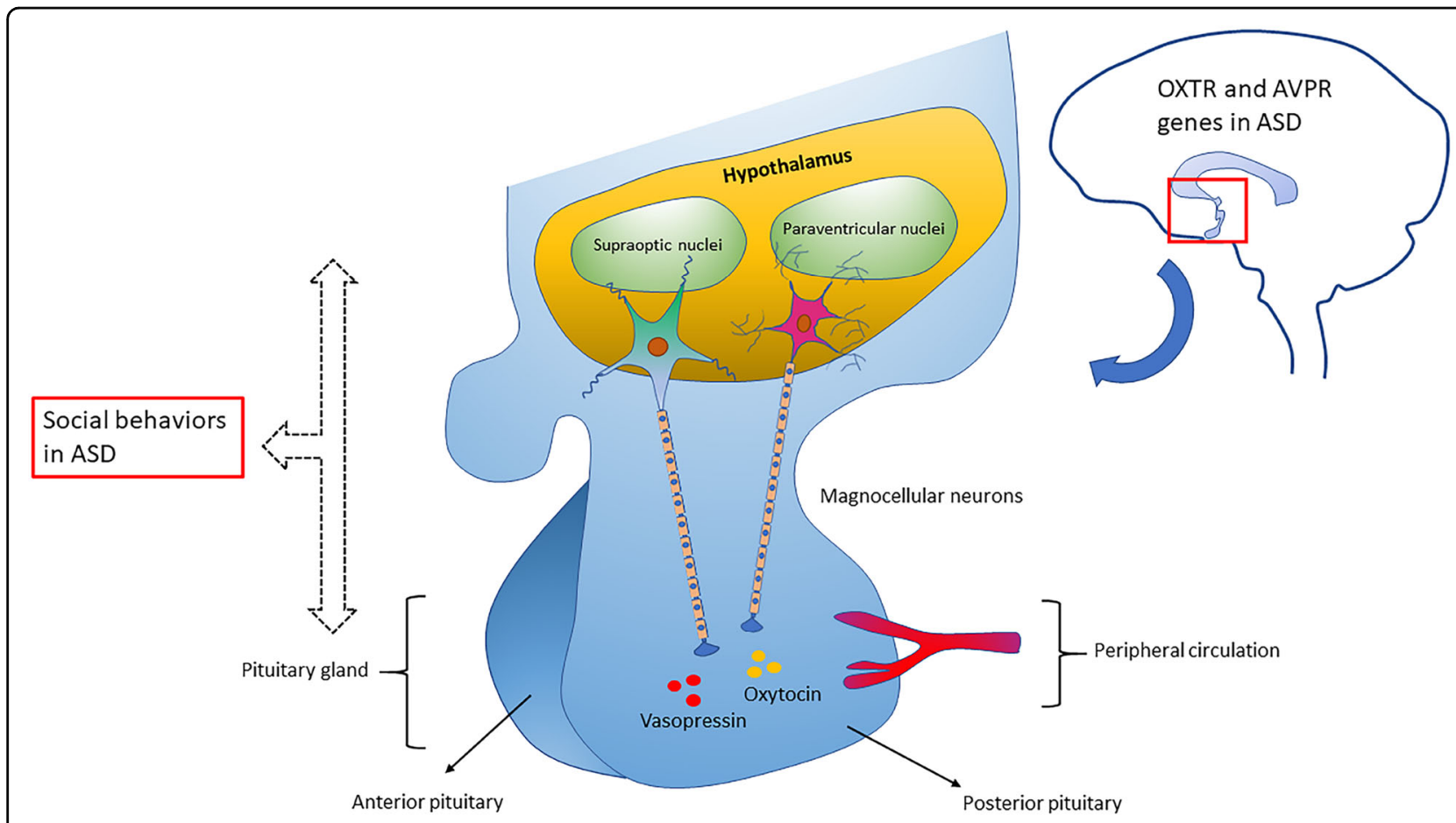

Fig. 3 Oxytocin and vasopressin (OT-AVP) pathway associated with social behavior in ASD. In the hypothalamus, magnocellular neurons originating from paraventricular and supraoptic nuclei release oxytocin and vasopressin in the posterior pituitary which then goes into the peripheral circulation. Any deficiencies in oxytocin or vasopressin levels contribute to ASD's social behavioral impairments.

neuron layering. Aberrant reelin signaling has been reported in many studies in individuals with $\mathrm{ASD}^{130-132}$. RELN mutations disrupt neuronal migration and connectivity and cerebellar hypoplasia ${ }^{133}$. A significant reduction in the RELN protein has also been observed in the cerebellum of autistic individuals ${ }^{132}$. Another gene $T$ Box Brain Protein 1 that encodes a brain-specific T-box transcription factor plays an essential role in the neuronal development, axonal migration, and the development of the cerebral cortex and amygdala ${ }^{134}$. This gene has been identified as an ASD causative gene ${ }^{135}$ and is involved in the differentiation of neurons during the early development of neocortex ${ }^{136}$.

\section{Diagnostic models of ASD}

\section{Diagnostic models based on MR-derived features}

MRI-based diagnostic models are used for the behavioral assessment of autistic patients. These diagnostic model studies involve three steps, including extraction of properties from MR images, construction of diagnostic model using statistical models followed by evaluation and validation by researchers. Several studies have focussed on MRIbased diagnostic models for the detection and classification of $\mathrm{ASD}^{30,137}$. Diagnostic model performance is strongly influenced by types of entities selected as components of the model. For example, a study showed the comparison of diagnostic models based on regional thickness derived from surface morphometry (SBM) with diagnostic models based on volumetric morphometry involving four different classification methods and classification based on thickness was found to be more efficient and predictive of ASD compared to classification based on volume ${ }^{30}$. rsfMRI pipelines have been used to extract predictive biomarkers in autism by constructing participant-specific connectomes and then comparing these connectomes across participants to learn connectivity patterns that may identify ASD individuals ${ }^{138}$. The results suggested that rsfMRI data collected from different sites could reveal robust functional connectivity biomarkers of ASD.

\section{Diagnostic models based on imaging genetics}

Imaging genetics in ASD has proven useful, and pathways that include common genetic variation in TD individuals at risk of developing ASD have been characterized. Prenatal transcription regulation and synapse formation in the developing brain is impacted by the genes associated with ASD (Table 1). Alteration in frontal WM connectivity and structure and disturbance in the frontal, temporal, and occipital circuits involved in visual and language processing was found to be associated with NRXN superfamily genes ${ }^{115}$. Neuropeptide signaling and emotional functioning was found to be influenced by the oxytocin and arginine vasopressin receptor genes via structural and functional modification in the 
amygdala-hypothalamus circuitry ${ }^{77}$. One study showed a relationship between frontal lobe connectivity and common genetic variants in CNTNAP2 using a functional neuroimaging study ${ }^{117}$ and the study found that ASD and TD individuals who were nonrisk allele carriers showed more reduction in the activation of $\mathrm{MPFC}$ during an fMRI task as compared to risk allele carriers ${ }^{117}$. Another study showed decreased functional connectivity in the prefrontal cortex, cortical spinal tract, corpus callosum, and decreased integrity of WM in children and adolescents carrying MET rs1858830, C risk allele. Such studies suggest that the genes affect the brain regions that are involved in social and emotional processing ${ }^{139}$.

More detailed brain analysis combined with visualization, will promote the elucidation of the relationship between genes and altered neuronal circuits. Also, researching the time-dependent impacts of ASD-involved genetic variants that are the cause of neurodevelopmental modification can aid in the progress of ASD imaging genetics. In addition, the plasticity that is altered in different neuropsychiatric disorders such as ASD can be measured with high resolution by combining electroencephalography or fMRI with transcranial magnetic stimulation. Advances in noninvasive brain imaging techniques and scans during rest or task may help in infant neuroimaging that are at a higher risk to develop ASD. With studies investigating genetic effects on brain development using high-resolution imaging, studying brain-behavior connections and the impact of new therapies on brain development, further translational progress will happen in the future. By neuroimaging endophenotypes, a greater understanding of the impact of ASD risk genes on the brain circuitry ${ }^{139}$ can be achieved. Ultimately, imaging genetics in ASD might be promising for the clinical management of ASD-affected patients.

\section{Challenges and future perspectives}

The field of imaging genetics has exponentially grown in recent decades from its candidate gene studies to largescale longitudinal studies, cross-modal investigations, and translational animal models of various psychiatric disorders. In addition, imaging genetics has begun integrating transcriptomic data and analytical methods for assessing pathway enrichment, such as the score system for pathway regulation. Of addition to translational animal research and pharmacological intervention in vitro and in vivo, imaging genetics can also provide an insight into various behavioral and genetic factors that contribute to the risk of ASDs.

One of the challenges facing imaging genetics is the conceptualization of endophenotypes, which states that endophenotypes are heritable and associated with psychiatric disorders and may impede research on brainbased associations by limiting imaging genetic research to genes previously associated with a psychiatric disorder ${ }^{140}$. It is important to properly replicate the studies, particularly those with false-positive results, to address the impact of a genetic variation in a disease, and this problem can be solved by correcting genome-wide associations with large sample size imaging phenotypes.

Another challenge in ASD neuroimaging research is the discrepancy in the brain connectivity findings due to motion artifacts. The motion artifacts in the rsfMRI data can misrepresent group differences in connectivity metrics which can lead to incorrect interpretation of the altered brain connectivity data in ASD. Other methodological variables such as pipeline type, field of view, and dataset can significantly impact (in terms of over-or underconnectivity) the functional connectivity MRI studies in $\mathrm{ASD}^{141}$. Also, the differences in age and severity of the ASD cohort may lead to disparity in findings, and so large-scale studies are required that concentrate on exploring the developmental trajectories that are crucial to understanding the neurobiology underlying ASD. Such large-scale study databases can be obtained from various consortia, such as autism brain imaging data exchange and National Autism Research Database (NDAR). The heterogeneity of the ASD phenotypes, therefore, leads to the difficulties faced in ASD neuroimaging genetics as there is a high degree of variability in frequency, the number of behavioral symptoms reported, and the associated characteristics in ASD. In addition, the brain responses produced as a result of a particular task stimulus are inadequate to determine if these responses result from a neural processing disorder or are due to the introduction of an extrinsic factor.

In addition, more development in data analytical methods such as machine learning algorithms or software can allow data from multiple modalities to be incorporated into a heterogeneous population, and can also recognize and detect digital biomarkers ${ }^{142}$ that can be used for the clinical management of ASD patients.

\section{Conclusion}

To explain the origin of ASD's underlying pathophysiology, the study of genetic alterations and the relationship between genes and environmental factors is crucial. Imaging genetics studies have revealed important information about the pathophysiology of ASD. One of the key aims of imaging genetics studies in ASD is to elucidate neural pathways that give rise to phenotypical heterogeneity. Enhanced risk of ASD is shown to be related to several different singlenucleotide polymorphisms, and the stratification of neuroimaging data by specific genetic risk factors occurring in ASD may help to understand the neurobiology underlying this disorder. Such studies will help to elucidate ASD from various perspectives and subsequently will pave the way for personalized treatment for patients with ASD. 


\section{Acknowledgements}

This study was supported by a PI grant from Sidra Medicine (5071012001) to M. H. A.A.B. is supported by Sidra Medicine internal grant (5011041002).

\section{Author details}

${ }^{1}$ Functional and Molecular Imaging Laboratory, Sidra Medicine, Doha, Qatar. ${ }^{2}$ Department of Psychiatry, Sidra Medicine, Doha, Qatar. ${ }^{3}$ Center for Magnetic Resonance and Optical Imaging, Department of Radiology, Perelman School of Medicine, University of Pennsylvania, Philadelphia, PA 19104, USA. ${ }^{4}$ Department of Human Genetics, Sidra Medicine, Doha, Qatar. ${ }^{5}$ Department of Genetic Medicine, Weill Cornell Medical College, Doha, Qatar. ${ }^{6}$ Academic Health System, Hamad Medical Corporation, Doha, Qatar. 'Laboratory Animal Research Center, Qatar University, Doha, Qatar

\section{Author contributions}

S.H., S.N, A.A.B.: prepared the scientific material, wrote the paper, generated tables and figures. S.Y., M.W., P.B., K.F., R.R., M.P.F.: critical revision and editing of the scientific contents. M.H. conceived of and designed the review contents, and contributed to paper writing and editing. All authors read and approved the final paper.

\section{Conflict of interest}

The authors declare that they have no conflict of interest.

\section{Publisher's note}

Springer Nature remains neutral with regard to jurisdictional claims in published maps and institutional affiliations.

Received: 20 February 2020 Revised: 5 June 2020 Accepted: 9 June 2020 Published online: 13 July 2020

\section{References}

1. Baio, J. et al. Prevalence of autism spectrum disorder among children aged 8 years - autism and developmental disabilities monitoring network, 11 sites, United States, 2014. MMWR Surveill. Summ. 67, 1-23 (2018).

2. Xu, G., Strathearn, L., Liu, B. \& Bao, W. Prevalence of autism spectrum disorder among US children and adolescents, 2014-2016. JAMA 319, 81-82 (2018).

3. Taylor, B., Jick, H. \& MacLaughlin, D. Prevalence and incidence rates of autism in the UK: time trend from 2004-2010 in children aged 8 years. BMJ Open 3, e003219 (2013).

4. Qiu, S. et al. Prevalence of autism spectrum disorder in Asia: a systematic review and meta-analysis. Psychiatry Res. 284, 112679 (2020).

5. Al-Farsi, Y. M. et al. Brief report: prevalence of autistic spectrum disorders in the Sultanate of Oman. J. Autism Dev. Disord. 41, 821-825 (2011).

6. Al-Ansari, A. \& Mahmood, M. Epidemiology of autistic disorder in Bahrain: prevalence and obstetric and familial characteristics. East. Mediterr. Health J. 19, 769-774 (2013)

7. Aljarallah, A., Alwaznah, T., Alansari, S. \& Alhazmi, M. A. Study of Autism and Developmental Disorders in Saudi Children (King Abdulaziz City for Science and Technology, Riyadh, Saudi Arabia, 2007).

8. Alshaban, F. et al. Prevalence and correlates of autism spectrum disorder in Qatar: a national study. J. Child Psychol. Psychiatry 60, 1254-1268 (2019).

9. Werling, D. M. \& Geschwind, D. H. Sex differences in autism spectrum disorders. Curr. Opin. Neurol. 26, 146-153 (2013).

10. Pisula, E. \& Porębowicz-Dörsmann, A. Family functioning, parenting stress and quality of life in mothers and fathers of Polish children with high functioning autism or Asperger syndrome. PLoS One 12, e0186536 (2017).

11. Gaugler, T. et al. Most genetic risk for autism resides with common variation. Nat. Genet. 46, 881-885 (2014).

12. Hallmayer, J. et al. Genetic heritability and shared environmental factors among twin pairs with autism. Arch. Gen. Psychiatry 68, 1095-1102 (2011).

13. Sandin, S. et al. The familial risk of autism. JAMA 311, 1770-1777 (2014).

14. O'Roak, B. J. et al. Sporadic autism exomes reveal a highly interconnected protein network of de novo mutations. Nature 485, 246-250 (2012).

15. De Rubeis, S. et al. Synaptic, transcriptional and chromatin genes disrupted in autism. Nature 515, 209-215 (2014).
16. Weiss, L. A. et al. A genome-wide linkage and association scan reveals novel loci for autism. Nature 461, 802-808 (2009).

17. Fakhoury, M. Imaging genetics in autism spectrum disorders: linking genetics and brain imaging in the pursuit of the underlying neurobiological mechanisms. Prog. Neuro-Psychopharmacol. Biol. Psychiatry 80, 101-114 (2018).

18. Kemper, T. L. \& Bauman, M. Neuropathology of infantile autism. J. Neuropathol. Exp. Neurol. 57, 645-652 (1998).

19. Courchesne, E. et al. Neuron number and size in prefrontal cortex of children with autism. JAMA 306, 2001-2010 (2011).

20. Arin, D. M., Bauman, M. L. \& Kemper, T. L. The distribution of Purkinje cell loss in the cerebellum in autism. Neurology 41, 307 (1991).

21. Bauman, M. L. \& Kemper, T. L. Neuroanatomic observations of the brain in autism: a review and future directions. Int. J. Dev. Neurosci. 23, 183-187 (2005).

22. Zikopoulos, B. \& Barbas, H. Changes in prefrontal axons may disrupt the network in autism. J. Neurosci. 30, 14595-14609 (2010).

23. Amaral, D. G., Schumann, C. M. \& Nordahl, C. W. Neuroanatomy of autism. Trends Neurosci. 31, 137-145 (2008).

24. Sparks, B. F. et al. Brain structural abnormalities in young children with autism spectrum disorder. Neurology 59, 184 (2002).

25. Nordahl, C. W. et al. Brain enlargement is associated with regression in preschool-age boys with autism spectrum disorders. Proc. Natl. Acad. Sci. USA 108, 20195-20200 (2011).

26. Courchesne, E. et al. Unusual brain growth patterns in early life in patients with autistic disorder. Neurology 57, 245 (2001).

27. Piven, J. \& An, M. R. I. study of the corpus callosum in autism. Am. J. Psychiatry 154, 1051-1056 (1997)

28. Hardan, A. Y. et al. study of increased cortical thickness in autism. Am. J. Psychiatry 163, 1290-1292 (2006).

29. Hyde, K. L., Samson, F., Evans, A. C. \& Mottron, L. Neuroanatomical differences in brain areas implicated in perceptual and other core features of autism revealed by cortical thickness analysis and voxel-based morphometry. Hum. Brain Mapp. 31, 556-566 (2010).

30. Jiao, Y. et al. Predictive models of autism spectrum disorder based on brain regional cortical thickness. Neurolmage 50, 589-599 (2010).

31. Libero, L. E., DeRamus, T. P., Deshpande, H. D. \& Kana, R. K. Surfacebased morphometry of the cortical architecture of autism spectrum disorders: volume, thickness, area, and gyrification. Neuropsychologia 62, 1-10 (2014).

32. Nordahl, C. W. et al. Cortical folding abnormalities in autism revealed by surface-based morphometry. J. Neurosci. 27, 11725-11735 (2007).

33. Chen, R., Jiao, Y. \& Herskovits, E. H. Structural MRI in autism spectrum disorder. Pediatr. Res. 69, 63R-68R (2011).

34. Mueller, S. et al. Convergent findings of altered functional and structural brain connectivity in individuals with high functioning autism: a multimodal MRI study. PLoS One 8, e67329 (2013).

35. Le Bihan, D. Looking into the functional architecture of the brain with diffusion MRI. Nat. Rev. Neurosci. 4, 469-480 (2003).

36. Travers, B. G. et al. Diffusion tensor imaging in autism spectrum disorder: a review. Autism Res. 5, 289-313 (2012).

37. Ben Bashat, D. et al. Accelerated maturation of white matter in young children with autism: a high b value DWI study. Neurolmage 37, 40-47 (2007).

38. Sahyoun, C. P., Belliveau, J. W. \& Mody, M. White matter integrity and pictorial reasoning in high-functioning children with autism. Brain Cognit. 73, 180-188 (2010).

39. Wolff, J. J. et al. Differences in white matter fiber tract development present from 6 to 24 months in infants with autism. Am. J. Psychiatry 169, 589-600 (2012).

40. Jou, R. J. et al. Diffusion tensor imaging in autism spectrum disorders: preliminary evidence of abnormal neural connectivity. Aust. N. Z. J. Psychiatry $\mathbf{4 5}$ 153-162 (2011).

41. Voineagu, I. et al. Transcriptomic analysis of autistic brain reveals convergent molecular pathology. Nature 474, 380-384 (2011).

42. Geschwind, D. H. \& State, M. W. Gene hunting in autism spectrum disorder: on the path to precision medicine. Lancet Neurol. 14, 1109-1120 (2015).

43. Kumar, $M$. et al. High resolution magnetic resonance imaging for characterization of the neuroligin-3 knock-in mouse model associated with autism spectrum disorder. PLoS One 9, e109872 (2014). 
44. Steadman, P. E. et al. Genetic effects on cerebellar structure across mouse models of autism using a magnetic resonance imaging atlas. Autism Res. 7, 124-137 (2014).

45. Schoen, $M$. et al. Shank3 transgenic and prenatal zinc-deficient autism mouse models show convergent and individual alterations of brain structures in MRI. Front. Neural Circuits 13, https://doi.org/10.3389/fncir.2019.00006 (2019)

46. Yang, R. et al. ANK2 autism mutation targeting giant ankyrin-B promotes axon branching and ectopic connectivity. Proc. Natl. Acad. Sci. USA 116, 15262-15271 (2019).

47. Lamar, K.-M. J. \& Carvill, G. L. Chromatin remodeling proteins in epilepsy: lessons from CHD2-associated epilepsy. Front. Mol. Neurosci. 11, 208-208 (2018).

48. Shen, T., Ji, F., Yuan, Z. \& Jiao, J. CHD2 is required for embryonic neurogenesis in the developing cerebral cortex. Stem Cells 33, 1794-1806 (2015).

49. Suls, A. et al. De novo loss-of-function mutations in CHD2 cause a feversensitive myoclonic epileptic encephalopathy sharing features with Dravet syndrome. Am. J. Hum. Genet. 93, 967-975 (2013).

50. Li, J. et al. Genes with de novo mutations are shared by four neuropsychiatric disorders discovered from NPdenovo database. Mol. Psychiatry 21, 290-297 (2016).

51. $\mathrm{Xu}, \mathrm{Q}$. et al. Autism-associated CHD8 deficiency impairs axon development and migration of cortical neurons. Mol. Autism 9, 65 (2018).

52. Sugathan, A. et al. CHD8 regulates neurodevelopmental pathways associated with autism spectrum disorder in neural progenitors. Proc. Natl. Acad. Sci. USA 111, E4468-E4477 (2014).

53. Rossel, M. \& Capecchi, M. R. Mice mutant for both Hoxa1 and Hoxb1 show extensive remodeling of the hindbrain and defects in craniofacial development. Development 126, 5027 (1999).

54. Qureshi, A. Y. et al. Opposing brain differences in 16p11.2 deletion and duplication carriers. J. Neurosci. 34, 11199-11211 (2014).

55. Bernier, R. et al. Disruptive CHD8 mutations define a subtype of autism early in development. Cell 158, 263-276 (2014).

56. Fang, W.-Q. et al. Overproduction of upper-layer neurons in the neocortex leads to autism-like features in mice. Cell Rep. 9, 1635-1643 (2014).

57. Richter, M. et al. Altered TAOK2 activity causes autism-related neurodevelopmental and cognitive abnormalities through RhoA signaling. Mol. Psychiatry 24, 1329-1350 (2019).

58. Qiu, S., Anderson, C. T., Levitt, P. \& Shepherd, G. M. G. Circuit-specific intracortical hyperconnectivity in mice with deletion of the autism-associated Met receptor tyrosine kinase. J. Neurosci. 31, 5855-5864 (2011).

59. Powell, E. M., Mars, W. M. \& Levitt, P. Hepatocyte growth factor/scatter factor is a motogen for interneurons migrating from the ventral to dorsal telencephalon. Neuron 30, 79-89 (2001).

60. Bassett, A. S. et al. Practical guidelines for managing patients with $22 \mathrm{q} 11.2$ deletion syndrome. J. Pediatr. 159, 332-339 (2011).

61. Moreno-De-Luca, A. et al. The role of parental cognitive, behavioral, and motor profiles in clinical variability in individuals with chromosome 16p11.2 deletions. JAMA Psychiatry 72, 119-126 (2015).

62. Mihailov, A. et al. Morphological brain changes associated with negative symptoms in patients with 22q11.2 deletion syndrome. Schizophr. Res. $\mathbf{1 8 8}$ 52-58 (2017).

63. Schmitt, J. E. et al. Aberrant cortical morphometry in the 22q11.2 deletion syndrome. Biol. Psychiatry 78, 135-143 (2015).

64. Fombonne, E., Rogé, B., Claverie, J., Courty, S. \& Frémolle, J. Microcephaly and macrocephaly in autism. J. Autism Dev. Disord. 29, 113-119 (1999).

65. Torres, M. \& Giráldez, F. The development of the vertebrate inner ear. Mech. Dev. 71, 5-21 (1998).

66. Persico, A. M. in Neural Circuit Development and Function in the Brain (eds Rubenstein, John L. R. \& Rakic, Pasko) 651-694 (Academic Press, Cambridge, 2013).

67. Muscarella, L. A. et al. HOXA1 gene variants influence head growth rates in humans. Am. J. Med. Genet. Part B: Neuropsychiatr. Genet. 144B, 388-390 (2007).

68. Conciatori, M. et al. Association between the HOXA1 A218G polymorphism and increased head circumference in patients with autism. Biol. Psychiatry $\mathbf{5 5}$ 413-419 (2004).

69. Muscarella, L. A. et al. Candidate gene study of HOXB1 in autism spectrum disorder. Mol. Autism 1, 9 (2010).
70. Pilarski, R. \& Eng, C. Will the real Cowden syndrome please stand up (again)? Expanding mutational and clinical spectra of the PTEN hamartoma tumour syndrome. J. Med. Genet. 41, 323-326 (2004).

71. Goffin, A., Hoefsloot, L. H., Bosgoed, E., Swillen, A. \& Fryns, J.-P. PTEN mutation in a family with Cowden syndrome and autism. Am. J. Med. Genet. 105, 521-524 (2001).

72. Butler, M. G. et al. Subset of individuals with autism spectrum disorders and extreme macrocephaly associated with germline PTEN tumour suppressor gene mutations. J. Med. Genet. 42, 318-321 (2005).

73. Buxbaum, J. D. et al. Mutation screening of the PTEN gene in patients with autism spectrum disorders and macrocephaly. Am. J. Med. Genet. B Neuropsychiatr. Genet. 144B, 484-491 (2007)

74. Strauss, K. et al. Recessive symptomatic focal epilepsy and mutant contactinassociated protein-like 2. N. Engl. J. Med. 354, 1370-1377 (2006).

75. Canali, G. et al. Genetic variants in autism-related CNTNAP2 impair axonal growth of cortical neurons. Hum. Mol. Genet. 27, 1941-1954 (2018).

76. de Jong, J. et al. Cortical overgrowth in a preclinical forebrain organoid model of CNTNAP2-associated autism spectrum disorder. (2019).

77. Tan, G. C. Y., Doke, T. F., Ashburner, J., Wood, N. W. \& Frackowiak, R. S. J. Normal variation in fronto-occipital circuitry and cerebellar structure with an autismassociated polymorphism of CNTNAP2. Neurolmage 53, 1030-1042 (2010).

78. Hedrick, A. et al. Autism risk gene MET variation and cortical thickness in typically developing children and adolescents. Autism Res. 5, 434-439 (2012).

79. Rudie, J. D. et al. Autism-associated promoter variant in MET impacts functional and structural brain networks. Neuron 75, 904-915 (2012).

80. Wassink, T. H. et al. Cerebral cortical gray matter overgrowth and functional variation of the serotonin transporter gene in autism. Arch. Gen. Psychiatry 64, 709-717 (2007).

81. Inoue, $H$. et al. Association between the oxytocin receptor gene and amygdalar volume in healthy adults. Biol. Psychiatry 68, 1066-1072 (2010).

82. Furman, D. J., Chen, M. C. \& Gotlib, I. H. Variant in oxytocin receptor gene is associated with amygdala volume. Psychoneuroendocrinology 36, 891-897 (2011).

83. Tost, H. et al. A common allele in the oxytocin receptor gene (OXTR) impacts prosocial temperament and human hypothalamic-limbic structure and function. Proc. Natl. Acad. Sci. USA 107, 13936-13941 (2010).

84. Saito, Y. et al. Neural correlate of autistic-like traits and a common allele in the oxytocin receptor gene. Soc. Cognit. Affect. Neurosci. 9, 1443-1450 (2014).

85. Damiano, C. R. et al. Association between the oxytocin receptor (OXTR) gene and mesolimbic responses to rewards. ecular. Mol. Autism 5, 7 (2014).

86. O'Connell, G. et al. Association of genetic variation in the promoter region of OXTR with differences in social affective neural processing. J. Behav. Brain Sci. 2, 60 (2012).

87. Montag, C., Sauer, C., Reuter, M. \& Kirsch, P. An interaction between oxytocin and a genetic variation of the oxytocin receptor modulates amygdala activity toward direct gaze: evidence from a pharmacological imaging genetics study. Eur. Arch. Psychiatry Clin. Neurosci. 263, 169-175 (2013).

88. Tost, H. et al. Neurogenetic effects of OXTR rs2254298 in the extended limbic system of healthy Caucasian adults. Biol. Psychiatry 70, e37-e39 (2011).

89. Schneider-Hassloff, $\mathrm{H}$. et al. Oxytocin receptor polymorphism and childhood social experiences shape adult personality, brain structure and neural correlates of mentalizing. Neurolmage 134, 671-684 (2016).

90. Marchetto, M. C. et al. Altered proliferation and networks in neural cells derived from idiopathic autistic individuals. Mol. Psychiatry 22, 820-835 (2017).

91. Olivito, G. et al. Resting-state functional connectivity changes between dentate nucleus and cortical social brain regions in autism spectrum disorders. Cerebellum 16, 283-292 (2017).

92. Joshi, G. et al. Integration and segregation of default mode network restingstate functional connectivity in transition-age males with high-functioning autism spectrum disorder: a proof-of-concept study. Brain Connect. 7 558-573 (2017).

93. Baddeley, A. Working memory: looking back and looking forward. Nat. Rev. Neurosci. 4, 829-839 (2003).

94. Seghier, M. L. The angular gyrus: multiple functions and multiple subdivisions. Neuroscientist 19, 43-61 (2013).

95. Abrams, D. A. et al. Underconnectivity between voice-selective cortex and reward circuitry in children with autism. Proc. Natl. Acad. Sci. USA 110, 12060-12065 (2013). 
96. Just, M. A., Cherkassky, V. L., Keller, T. A. \& Minshew, N. J. Cortical activation and synchronization during sentence comprehension in high-functioning autism: evidence of underconnectivity. Brain 127, 1811-1821 (2004).

97. Koshino, $\mathrm{H}$. et al. Functional connectivity in an $\mathrm{fMRI}$ working memory task in high-functioning autism. Neurolmage 24, 810-821 (2005).

98. Kleinhans, N. M. et al. Abnormal functional connectivity in autism spectrum disorders during face processing. Brain 131, 1000-1012 (2008).

99. Kana, R. K., Keller, T. A., Minshew, N. J. \& Just, M. A. Inhibitory control in highfunctioning autism: decreased activation and underconnectivity in inhibition networks. Biol. Psychiatry 62, 198-206 (2007).

100. Villalobos, M. E., Mizuno, A., Dahl, B. C., Kemmotsu, N. \& Müller, R.-A. Reduced functional connectivity between $\mathrm{V} 1$ and inferior frontal cortex associated with visuomotor performance in autism. Neurolmage 25, 916-925 (2005).

101. Blakemore, S.J. The social brain in adolescence. Nat. Rev. Neurosci. 9, 267-277 (2008).

102. Kana, R. K. \& Wadsworth, H. M. "The archeologist's career ended in ruins": hemispheric differences in pun comprehension in autism. Neurolmage $\mathbf{6 2}$ 77-86 (2012)

103. Catarino, A. et al. An fMRl investigation of detection of semantic incongruities in autistic spectrum conditions. Eur. J. Neurosci. 33, 558-567 (2011).

104. Dichter, G. S. Functional magnetic resonance imaging of autism spectrum disorders. Dialog. Clin. Neurosci. 14, 319-351 (2012).

105. Ameis, S. H. \& Szatmari, P. Imaging-genetics in autism spectrum disorder advances, translational impact, and future directions. Front. Psychiatry 3, 46 (2012)

106. Noonan, S. K., Haist, F. \& Müller, R.-A. Aberrant functional connectivity in autism: evidence from low-frequency BOLD signal fluctuations. Brain Res. 1262, 48-63 (2009).

107. Safar, K., Wong, S. M., Leung, R. C., Dunkley, B. T. \& Taylor, M. J. Increased functional connectivity during emotional face processing in children with autism spectrum disorder. Front. Hum. Neurosci. 12, 408 (2018).

108. Martucci, L. L. et al. A multiscale analysis in CD38(-/-) mice unveils major prefrontal cortex dysfunctions. FASEB J. 33, 5823-5835 (2019).

109. Egashira, N. et al. Impaired social interaction and reduced anxiety-related behavior in vasopressin V1a receptor knockout mice. Behav. Brain Res. 178 123-127 (2007).

110. Bielsky, I. F., Hu, S.-B., Szegda, K. L., Westphal, H. \& Young, L. J. Profound impairment in social recognition and reduction in anxiety-like behavior in vasopressin V1a receptor knockout mice. Neuropsychopharmacology 29, 483-493 (2004).

111. Trommsdorff, M. et al. Reeler/disabled-like disruption of neuronal migration in knockout mice lacking the VLDL receptor and ApoE receptor 2. Cell 97, 689-701 (1999).

112. Salinger, W., Ladrow, P. \& Wheeler, C. Behavioral phenotype of the reeler mutant mouse: effects of reln gene dosage and social isolation. Behav. Neurosci. 117, 1257 (2004).

113. Domínguez-Iturza, N. et al. The autism- and schizophrenia-associated protein CYFIP1 regulates bilateral brain connectivity and behaviour. Nat. Commun. 10, 3454 (2019).

114. Liska, A. et al. Homozygous loss of autism-risk gene CNTNAP2 results in reduced local and long-range prefrontal functional connectivity. Cold Spring Harbor Labs J. 060335. https://doi.org/10.1101/060335. (2016).

115. Voineskos, A. N. et al. Neurexin-1 and frontal lobe white matter: an overlapping intermediate phenotype for schizophrenia and autism spectrum disorders. Plos One 6, e20982 (2011).

116. Lam, M. et al. Single cell analysis of autism patient with bi-allelic NRXN1-alpha deletion reveals skewed fate choice in neural progenitors and impaired neuronal functionality. Exp. Cell Res. 383, 111469 (2019).

117. Scott-Van Zeeland, A. A. et al. Altered functional connectivity in frontal lobe circuits is associated with variation in the autism risk gene CNTNAP2. Sci. Transl. Med. 2, $56 \mathrm{ra} 80$ (2010).

118. Whalley, H. C. et al. Genetic variation in CNTNAP2 alters brain function during linguistic processing in healthy individuals. Am. J. Med. Genet. Part B: Neuropsychiatr. Genet. 156, 941-948 (2011).

119. Sauer, C., Montag, C., Wörner, C., Kirsch, P. \& Reuter, M. Effects of a common variant in the CD38 gene on social processing in an oxytocin challenge study: possible links to autism. Neuropsychopharmacology 37, 1474-1482 (2012).

120. Meyer-Lindenberg, A. et al. Genetic variants in AVPR1A linked to autism predict amygdala activation and personality traits in healthy humans. Mol. Psychiatry 14, 968-975 (2009).
121. Sauer, C., Montag, C., Reuter, M. \& Kirsch, P. Imaging oxytocin $\times$ dopamine interactions: an epistasis effect of CD38 and COMT gene variants influences the impact of oxytocin on amygdala activation to social stimuli. Front Neurosci. 7, 45 (2013).

122. Liska, A., Galbusera, A., Schwarz, A. J. \& Gozzi, A. Functional connectivity hubs of the mouse brain. Neurolmage 115, 281-291 (2015).

123. de Oliveira Pereira Ribeiro, L. et al. Evidence for association between OXTR gene and ASD clinical phenotypes. J. Mol. Neurosci. 65, 213-221 (2018).

124. Boso, M. et al. Reduced plasma apelin levels in patients with autistic spectrum disorder. Arch. Med. Res. 38, 70-74 (2007).

125. Tansey, K. E. et al. Functionality of promoter microsatellites of arginine vasopressin receptor 1 A (AVPR1A): implications for autism. Mol. Autism 2, 3 (2011).

126. Muma, N. A. \& Hoffman, P. N. Neurofilaments are intrinsic determinants of axonal caliber. Micron 24, 677-683 (1993).

127. Smorodchenko, A. et al. Comparative analysis of uncoupling protein 4 distribution in various tissues under physiological conditions and during development. Biochim. Biophys. Acta-Biomembr. 1788, 2309-2319 (2009).

128. Zhang, M. et al. Overexpression of uncoupling protein 4 promotes proliferation and inhibits apoptosis and differentiation of preadipocytes. Life Sci. 79, 1428-1435 (2006).

129. Liu, D. et al. Mitochondrial UCP4 mediates an adaptive shift in energy metabolism and increases the resistance of neurons to metabolic and oxidative stress. NeuroMol. Med. 8, 389-413 (2006).

130. Bonora, E. et al. Analysis of reelin as a candidate gene for autism. Mol. Psychiatry 8, 885-892 (2003).

131. Persico, A. M. et al. Reelin gene alleles and haplotypes as a factor predisposing to autistic disorder. Mol. Psychiatry 6, 150-159 (2001).

132. Fatemi, S. H. et al. Reelin signaling is impaired in autism. Biol. Psychiatry 57, 777-787 (2005).

133. Hong, S. E. et al. Autosomal recessive lissencephaly with cerebellar hypoplasia is associated with human RELN mutations. Nat. Genet. 26, 93-96 (2000).

134. Huang, T.-N. \& Hsueh, Y.-P. Brain-specific transcriptional regulator T-brain-1 controls brain wiring and neuronal activity in autism spectrum disorders. Front. Neurosci. 9, 406 (2015).

135. Huang, T.-N. et al. Haploinsufficiency of autism causative gene Tbr1 impairs olfactory discrimination and neuronal activation of the olfactory system in mice. Mol. Autism 10, 5 (2019).

136. Englund, C. et al. Pax6, Tbr2, and Tbr1 are expressed sequentially by radial glia, intermediate progenitor cells, and postmitotic neurons in developing neocortex. J. Neurosci. 25, 247-251 (2005).

137. Ecker, $C$. et al. Describing the brain in autism in five dimensions-magnetic resonance imaging-assisted diagnosis of autism spectrum disorder using a multiparameter classification approach. J. Neurosci. 30, 10612-10623 (2010).

138. Abraham, A. et al. Deriving reproducible biomarkers from multi-site resting state data: an Autism-based example. Neurolmage 147, 736-745 (2017).

139. Hernandez, L. M., Rudie, J. D., Green, S. A., Bookheimer, S. \& Dapretto, M. Neural signatures of autism spectrum disorders: insights into brain network dynamics. Neuropsychopharmacology 40, 171-189 (2015).

140. Bogdan, R. et al. Imaging genetics and genomics in psychiatry: a critical review of progress and potential. Biol. Psychiatry 82, 165-175 (2017).

141. Nair, A. et al. Impact of methodological variables on functional connectivity findings in autism spectrum disorders. Hum. Brain Mapp. 35. https://doi.org/ 10.1002/hbm.22456 (2014).

142. Coravos, A., Khozin, S. \& Mandl, K. D. Developing and adopting safe and effective digital biomarkers to improve patient outcomes. NPJ Digit. Med. 2, 14 (2019).

143. Domínguez-del-Toro, E. et al. Generation of a novel functional circuit in Hoxa1 mutant mice. J. Neurosci. 21, 5637-5642 (2001).

144. Lazaro, M. T. et al. Reduced prefrontal synaptic connectivity and disturbed oscillatory population dynamics in the CNTNAP2 model of autism. Cell Rep. 27, 2567-2578 (2019).

145. Wang, C., Pan, Y.-H., Wang, Y., Blatt, G. \& Yuan, X.-B. Segregated expressions of autism risk genes Cdh11 and Cdh9 in autism-relevant regions of developing cerebellum. Mol. Brain 12, 40 (2019).

146. Lepagnol-Bestel, A. M. et al. SLC25A12 expression is associated with neurite outgrowth and is upregulated in the prefrontal cortex of autistic subjects. Mol. Psychiatry 13, 385-397 (2008).

147. Lintas, C. et al. Involvement of the PRKCB1 gene in autistic disorder: significant genetic association and reduced neocortical gene expression. Mol. Psychiatry 14, 705-718 (2009). 
148. Nagarajan, R. P., Hogart, A. R., Gwye, Y., Martin, M. R. \& LaSalle, J. M. Reduced MeCP2 expression is frequent in autism frontal cortex and correlates with aberrant MECP2 promoter methylation. Epigenetics 1, e1-e11 (2006).
149. Xing, X. et al. Hyperactive Akt-mTOR pathway as a therapeutic target for pain hypersensitivity in Cntnap2-deficient mice. Neuropharmacology 165, 107816 (2020). 